\title{
Les Grands Mammifères du Burdigalien (MN3; Miocène) d'Estrepouy (Gers, France): liste faunique actualisée
}

\section{The Early Burdigalian (MN3; Miocene) large mammals from Estrepouy (Aquitaine basin, France): an updated faunal list}

\author{
L. Ginsburg 1
}

\section{RÉSUMÉ}

Des grands mammifères sont determines pour le Miocène inférieur (MN3) de Etrepouy, Gers, France. 17 taxons appartenant à trois déterminés ont été identifies: Carnivora; Amphicyon lanthanicus, Cynelos helbingi, Plithocyon bruneti, Hemicyon gargan, Palaeogale hyaenoides, Semigenetta elegans et Pseudaelurus turnauensis. Perissodactyla; Anchitherium aurelianense, Protaceratherium minutum et Diaceratherium cf. aurelianense. Arctiodactyla; Aureliachoerus aurelianensis, Xenohyus venitor, Caenotherium aff. lintillae, Andegameryx andegaviensis, Oriomeryx willii, Procervulus praelucidus, Lagomeryx parvulus et Procervulus praelucidus. L'association des mammifères Estrepouy regarde un peu plus âgé que celui représenté à la localité de référence du MN3 á Wintershof-Ouest (Allemagne).

Mots clés: Mammalia, Carnivora, Perissodactyla, Artiodactyla, Estrepouy, France, Miocene inferiour

\begin{abstract}
The purpose of the present work is to describe the mammals from the Early Miocene locality of Estrepouy, Gers, France. We have identified 17 species belonging to 3 orders; Carnivora; Amphicyon lanthanicus, Cynelos helbingi, Plithocyon bruneti, Hemicyon gargan, Palaeogale hyaenoides, Semigenetta elegans y Pseudaelurus turnauensis. Perissodactyla; Anchitherium aurelianense, Protaceratherium minutum y Diaceratherium cf. aurelianense. Arctiodactyla; Aureliachoerus aurelianensis, Xenohyus venitor, Caenotherium aff. lintillae, Andegameryx andegaviensis, Oriomeryx willii, Procervulus praelucidus, Lagomeryx parvulus y Procervulus praelucidus. The Estrepouy mammal assemblage seems older than that represented in Wintershof-West (Alemania), MN 3 reference locality.
\end{abstract}

Keywords: Mammalia, Carnivora, Perissodactyla, Artiodactyla, Estrepouy, France, Lower Miocene

\section{Introduction}

Le gisement d'Estrepouy (Gers) fut découvert par Charles Cadéot, vétérinaire dans la région et auteur de nombreuses découvertes de gisements; ses trouvailles furent communiquées à C. Depéret qui entreprit des fouilles avec ses collaborateurs F. Roman et
J. Viret. Après le décès de Depéret, les travaux furent continués et une coupe ainsi qu'une étude de la faune du gisement d'Estrepouy furent publiés par Roman et Viret (1930), (1934).

La présence du genre Anchitherium et des premiers cervidés à bois justifient le rattachement du gisement d'Estrepouy au Orleaniense. La localité

\footnotetext{
1 Avertissement: Léonard Ginsburg (1927-2009) a laissé inachevé, mais à un stade très avancé, un manuscrit sur la faune de grands mammifères d'Estrepouy. Dépositaire de ce travail, Marguerite Hugueney m'a suggéré de l'actualiser afin de donner une vision d'ensemble de l'importante faune d'Estrepouy. Ayant révisé le manuscrit, et estimant que la détermination des taxons était rigoureuse, j'ai préféré ne pas modifier le texte et le laisser dans son état original, en dépit de son caractère provisoire. Le texte est donc entièrement de Léonard Ginsburg, les références du manuscrit et la figuration de quelques pièces décrites ont été ajoutées par moi. De manière indépendante, et afin de compléter ce travail, Sanisidro (2011) a décrit dans le présent volume quelques restes de rhinocéros conservés dans le Département des Sciences de la Terre (Université Claude Bernard, Lyon) qui n'avaient pas été inclus dans le manuscrit mentionné et Quiralte \& Morales (2011), dans ce volume également, ont décrit les fossiles déterminés comme Andegameryx andegaviensis. Jorge Morales. Madrid, le 18 novembre 2011.
} 
représentative de $\mathrm{MN} 3$, Wintershof-West (Bavière, Allemagne), est un peu plus récente.

Le matériel récolté à Estrepouy se trouve dans de nombreuses collections, aussi bien publiques (coll. Lyon $=$ FSL, coll. Paris $=$ MNHN, coll. Museum de Bâle $=\mathrm{GB}$, coll Bordeaux $/$ Ringeade $=\mathrm{R}$ ) que privées (coll. Bulot $=B$, coll. Harène $=H$ ); il est possible qu'une petite différence d'âge existe entre les différentes lentilles sableuses et par conséquent, entre les faunes récoltées.

\section{Etude systematique}

Ordre Carnivora Bowdich, 1821

Famille Amphicyonidae Haeckel, 1866

Genre Amphicyon Lartet, 1831

Amphicyon lathanicus Ginsburg, 2000

Matériel: i3d (coll.H) $=7$ x 10,3

Discussion: cette incisive, bien conservée, comporte une pointe centrale proéminente flanquée de deux pointes latérales plus basses, une mésiale et une distale. Cette dent est identique morphologiquement à la i3 du squelette complet d'Amphicyon major de Sansan récolté par Bergougnioux et Crouzel (1973). Sa taille correspond à ce que l'on pouvait attendre pour $A$. lathanicus. La famille avait été signalée dans le gisement par Viret à partir « d'un médiocre matériel».

Genre Cynelos Jourdan, 1848 (Gervais, 1848-1852) Cynelos helbingi (Dehm, 1950)

Matériel: p3g (FSL 320.339) = 9,45 x 4,1. Figs. 1A-B. p4d, partie antérieure $($ FSL 320.339) $=$ ?x 5,8. Figs. 2A-B.

Discussion: ces deux prémolaires s'accordent parfaitement au C. helbingi de Wintershof-West.

Famille Ursidae Fischer von Waldheim, 1817

Genre Plithocyon Ginsburg, 1955

Plithocyon bruneti Ginsburg, 1980

Matériel: P4 d (coll. H) =12,9 x 8,5

Genre Hemicyon Lartet, 1851

Hemicyon gargan Ginsburg et Morales, 1998

Matériel: m1d, talonide (FSL 320.339) 1 talonide =11,4. Fig.3.

Famille Mustelidae Fischer von Waldheim, 1817

Genre Palaeogale von Meyer, 1846

Palaeogale hyaenoides Dehm, 1950

1974 - Palaeogale minuta (Gervais) - Ginsburg, p. 159.

\section{Matériel: $\mathrm{M} 1 \mathrm{~g}=2,45$ x 5,1}

Discussion: cette petite tuberculeuse est pratiquement identique à la M1 de Wintershof-West faisant partie de l'hypodigme de la population-type de l'espèce (Dehm, 1950, n¹3.906, fig. 148).
Famille Viverridae Gray, 1821

Genre Semigenetta Helbing, 1927

Semigenetta elegans Dehm, 1950

1930 -Semigenetta repelini Helbing - Roman \& Viret p. 589

1974 - Semigenetta cf. repelini Helbing - Ginsburg, p. 159.

Matériel: m1d (FSL 320.354) = 7,2 x 3,4.Figs. 4A-C.

Famille Felidae Fischer von Waldheim, 1817

Genre Pseudaelurus Gervais, 1850

Pseudaelurus turnauensis (Hoernes, 1882)

Matériel: $\mathrm{I} 3 \mathrm{~d}=3,35 \mathrm{x} 2,5 \mathrm{P} 4 \mathrm{~g}$ incomplète (coll.H) L métastyle $=5,2$

Ordre Perissodactyla Owen, 1848

Famille Equidae Gray, 1821

Género Anchitherium von Meyer, 1834

Anchitherium aurelianense (Cuvier, 1825)

1930 Anchitherium aurelianensis - Roman \& Viret p. 589

Matériel: 1/2 P4d (FSL 320.330) 1 moitié postérieure = 22,2. Figs. 5A-B.

Discussion: la dent jugale supérieure est la pièce la plus complète; signalée par Roman \& Viret (1930), elle n'a jamais été figurée bien qu'elle soit la plus ancienne dent d'Anchitherium trouvée en Europe. Il semble que ce soit une P4 pour les raisons conjuguées suivantes: mésostyle très pincé, paroi labiale du métacône et importance du cingulum labial identiques aux P4 d' A. aurelianense de Baigneaux-en-Beauce (MN3b), hypocône non parfaitement cônique lingualement mais montrant une petite crête descendant lingualement de la pointe de l'hypocône; cette posthypocrista, nette sur les P3/4 des gisements les plus anciens (Estrepouy, Chilleurs-aux-Bois, Baigneaux) régresse ensuite pour n'être plus qu'occasionnelle dans les gisements plus récents (Sandelzhausen, Steinheim: Abusch-Sievert, (1983). Nous ajouterons que sur la dent d'Estrepouy, d'une part le métalophe ne dessine pas un $\mathrm{S}$ régulier mais deux arcs se réunissant par leur pointe, d'autre part la posthypocrista est d'orientation plus postérieure que linguale par rapport aux prémolaires connues de Chilleurs-aux-Bois et de Baigneaux-en Beauce. Ces deux caractères paraissent devoir être des caractères primitifs.

La P4 d'Estrepouy indique un sujet plus grand que ceux représentés à Chilleurs-aux-Bois et Baigneaux. Hernandez Fernandez et al. (2003) ont montré, qu'en Espagne, d'importantes variations de températures saisonnières pouvaient provoquer une augmentation de la taille des dents.

Famille Rhinocerotidae Gray, 1821

Genre Protaceratherium Abel, 1910

Protaceratherium minutum (Cuvier, 1824) 1930 Rhinoceros sp. - Roman \& Viret p. 588

Matériel: DP1d (coll. Bâle, GB 1824) = 18 x 12

Description et discussion: cette petite prémolaire lactéale supérieure est fine, étroite, allongée et dépourvue de toute 


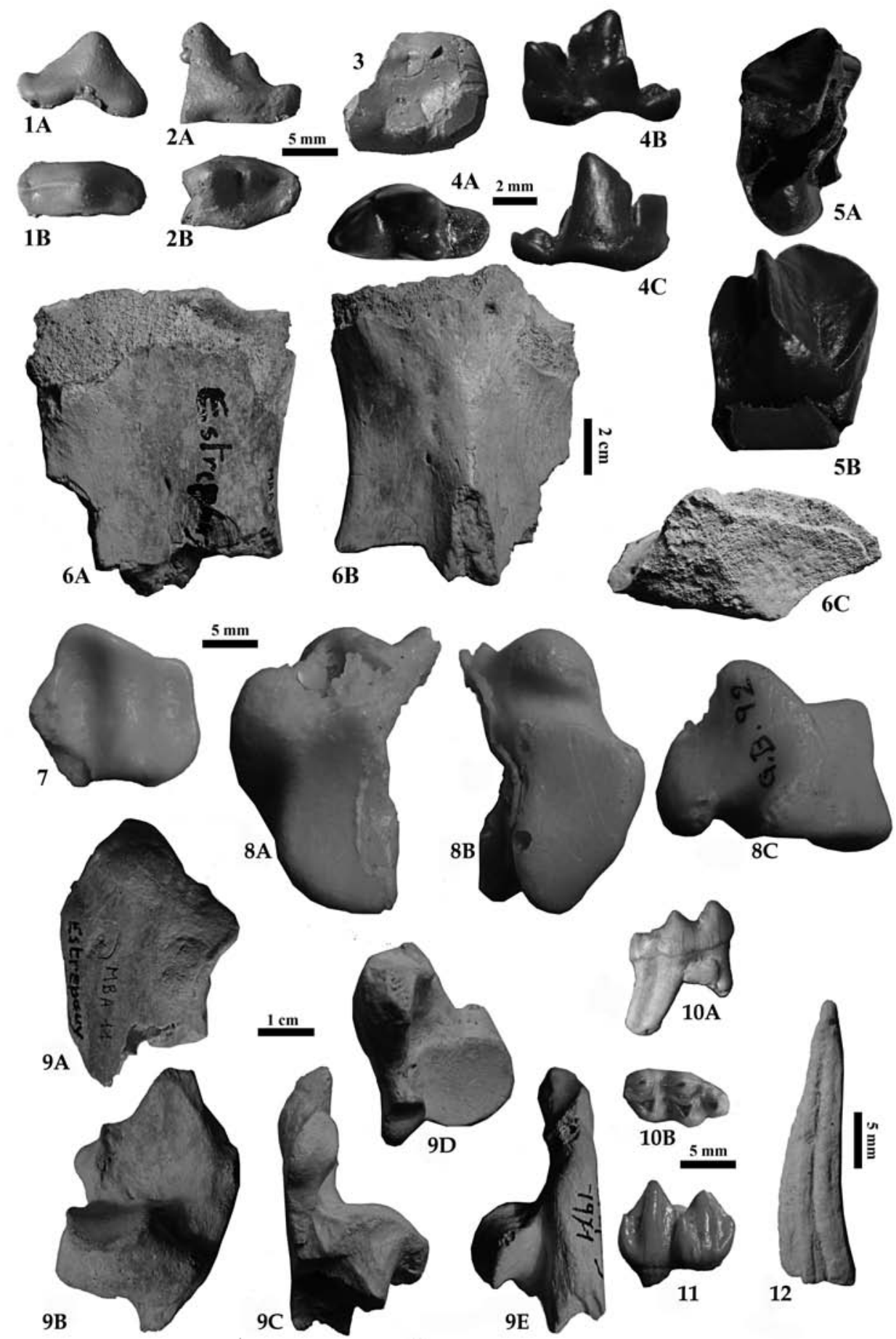

Fig. 1-12.-[1-2]: Cynelos helbingi (Dehm, (1950), 1) p3g (FSL 320.339): A. vue linguale; B. vue occlusale. 2) p4d, partie antérieure (FSL 320.339): A. vue linguale; B. vue occlusale. [3]: Hemicyon gargan Ginsburg \& Morales, (1998). m1d, talonide (FSL 320.339) vue occlusale. [4]: Semigenetta elegans Dehm, (1950). m1d (FSL 320.354) A. Vue occlusale: B. Vue linguale; C. Vue labiale. [5]: Anchitherium aurelianense (Cuvier, (1825)- moitié postérieure de P4d (FSL 320.330). A. vue occlusale; B. vue labiale. [6]: Diaceratherium cf. aurelianense (Nouel, (1866). omoplate g distale (GER 751. MNHN) A. Vue mediale; B. Vue laterale; C. Vue distale. [7]: Aureliachoerus aurelianensis (Stehlin, (1900) tibia d, extrémité distale (coll. Bâle, GB 989). Vue distale. [8]: Xenohyus venitor Ginsburg, (1980) cuboïde d (coll. Bâle, GB 92) incomplet à l'arrière. A. Vue proximale; B. Vue distale; C. Vue laterale. [9]: Oriomeryx willii Ginsburg, (1985). calcanéum g privé du manubrium (GER 750. MNHN). A. Vue laterale; B. Vue mediale; C. Vue plantaire; D. Vue proximale; E. Vue dorsale. [10]: Lagomeryx parvulus (Roger, (1898) m3g (FSL 320 154, figurée in Roman et Viret (1934), pl.V, fig. 29). A.Vue linguale; B. Vue occlusale. [11-12]: Procervulus praelucidus (Obergfell, (1957). 11. Muraille labiale de M2 g. 
usure. Le parastyle, le paracône et le métacône sont fondus ensemble en une longue crête qui occupe presque toute la dent. Ces trois éléments ne se distinguent que par la légère pointe qui émerge de la crête et marque le paracône et le métacône, ainsi que par la muraille externe qui est trilobée; cette trilobation indique un développement sensiblement égal des trois cuspides. Du côté lingual, la crête principale émet deux petites crêtes transversales qui correspondent au protolophe et au métalophe; le protolophe est relié à une petite crête longitudinale d'où émerge le protocône; le métalophe est relié à l'hypocône qui est rond et placé juste en arrière de la postprotocrista. Cette dent a été déterminée comme une DP1 de Protaceratherium minutum en raison de sa forte ressemblance morphologique et dimensionnelle avec une DP1 de Selles-sur-Cher où cette espèce est abondamment représentée.

\section{Genre Diaceratherium Dietrich, 1931 Diaceratherium cf. aurelianense (Nouel, 1866)}

Matériel: omoplate g distale (GER 751. MNHN) DAP au col =68,2. Cavité glénoïdienne: DT>50; DAP>90. Figs. 6A-C.

Description et discussion: L'os récolté est en mauvais état. C'est un os très plat. La cavité glénoïdienne est aplatie et nettement plus longue que large. L'épine scapulaire démarre assez loin du bord de la cavité glénoïdienne. Le tubercule supraglénoïdien est fort et séparé de la cavité glénoïdienne par un ensellement relativement large. Tous ces caractères se retrouvent sur les omoplates de Diaceratherium, en particulier celle du squelette presque complet $(\mathrm{Ar}(2160)$ de $D$. aurelianense récoltée en (1964) à Artenay et plusieurs récoltées isolément à Baigneauxen-Beauce; la taille est aussi la même. Il s'agit donc bien d'un Diaceratherium. L'omoplate de $D$. aginense de Laugnac figurée par Répelin (1917), pl.VI, fig.1) est bien voisine, mais le tubercule supraglénoïdien est bien plus fort et le fond de la cavité glénoïdienne beaucoup plus courbe en vue dorsale. Aussi nous rapprocherons la scapula d'Estrepouy de la forme de l'Orléanais.

Ordre Artiodactyla Owen, 1848

Famille Suidae Gray, 1821

Genre Aureliachoerus Ginsburg, 1974

Aureliachoerus aurelianensis (Stehlin, 1900) 1930 - Palaeochoerus aurelianensis Stehlin Roman \& Viret, p. 589.

Matériel: P2g (coll.B) = 8,3 x 4,7; M3d (coll. Bâle GB 1825) $=15,5 \times 12,2 ; \mathrm{dp} 1 \mathrm{~g}($ coll. $\mathrm{H})=7,45 \times 2,9$ tibia d, extrémité distale (coll. Bâle, GB 989) DT =15,7; DAP = 14,4. Fig. 7.

Discussion: La M3 et l'extrémité distale de tibia du museum de Bâle sont identiques à des pièces équivalentes de l'Aureliachoerus aurelianensis d'Artenay, où l'espèce est abondamment représentée.

La petite dent récoltée par Harène (dp1) est étroite, uniradiculée, légèrement bombée labialement et sa pointe unique est en position antérieure; de cette pointe descend vers l'arrière une fine crête en position labiale; une autre crête plus discrète la double à mi-hauteur du côté lingual et se termine par un bourrelet cingulaire distal. On ne connaît pas de p1 d'Aureliachoerus aurelianensis en place sur une mandibule mais plusieurs mandibules d'Artenay avec p4-m3 en place montrent l'alvéole de p1. L'étroitesse de la racine et la minceur de l'émail de la dent récoltée par Harène nous incitent à la considérer comme une dent lactéale.

La dent récoltée par Bulot est petite, allongée et comporte une pointe principale basse, en position antérieure, suivie d'une deuxième pointe plus basse et d'un petit cingulum bombé en position linguale. Cette dent correspond à la P2 du crâne d' Aureliachoerus aurelianensis trouvé à La Romieu par Collier \& Guex (1977), fig.1.

Famille Tayassuidae Palmer, 1897

Genre Xenohyus Ginsburg, 1980

Xenohyus venitor Ginsburg, 1980

Matériel: i3g = 6,55 x 9,5 cuboïde d (coll. Bâle, GB 92) incomplet à l'arrière. $\mathrm{L}$ face antérieure $=18,3$; DT partie antérieure $=14,5 ; \mathrm{DAP}=26$. Figs. 8 A-C.

Discussion: ce cuboïde, typiquement suiforme par sa morphologie, a été attribué à cette espèce en raison de sa taille.

Famille Cainotheriidae Cope, 1887

Genre Cainotherium Bravard, 1828

Caenotherium aff. lintillae Baudelot \& Crouzel, 1974

1930 - Cainotherium sp. - Roman \& Viret p. 589

1934 - Caenotherium sp. - Roman \& Viret, p.45.

Matériel: fragment de mand. g avec p2-p4 (FSL 32014 9, figurée in Roman \& Viret, (1934), pl.V, fig. 24); fragment de mand. g avec m2-m3 (FSL 32015 0, figurée in Roman \& Viret, (1934), pl.V, fig. 25); M sup. (FSL 32015 2, 32015 3, figurées in Roman \& Viret, (1934), pl.V, fig. 27-28).

Famille Andegamerycidae Ginsburg \& Morales, 1989

Genre Andegameryx Ginsburg, 1971

Andegameryx andegaviensis Ginsburg, 1971 1934 - Amphitragulus aurelianensis Mayet Roman \& Viret, p. 48

Matériel: P2g (FSL 320.199), figurée in Roman \& Viret (1934), pl.VII, fig. 15) = 8,9 x 5,3; M1g (FSL 320.200), figurée in Roman \& Viret (1934), pl.VII, fig. 16) = 9,8 x 11,05; m1d (FSL 320.197), figurée in Roman \& Viret (1934), pl.VII, fig. 13) $=9,3 \times 6,4 ; \mathrm{m} 2 \mathrm{~d}$ (FSL 320.198), figurée in Roman \& Viret (1934), pl.VII, fig.14) = 10,9 x 7,1; P3(?)g incomplète à l'angle antéro-labial (coll. Bulot) $=9 \times 7 ; \mathrm{M} 1 / 2 \mathrm{~g}($ coll. Bulot $)=$ 11,4 5 x 12,45; M1/2 (coll. Bâle, GB 993) = 9,3 x 6,1; m3d $($ coll. Bulot $)=15,4 \times 8$

Description et discussion: $\mathrm{P} 2$ est une dent à la fois usée et incomplète; elle est allongée et relativement étroite; un fort sillon vertical sépare, sur la muraille labiale, le métacône du métastyle; au pied du métastyle, cette muraille labiale est ornée de deux minuscules tubercules accessoires; de telles formations sont fréquentes sur les P3 et non les P2 d'A. laugnacensis de Grépiac (MN2a); aussi nous déterminerons cette dent d'Estrepouy comme une P3.

Les deux molaires supérieures sont des dents subcarrées à quatre tubercules; le parastyle et le mésostyle sont forts, le 
métastyle plus effacé; la protocrista ne rejoint pas la prohypocrista, qui est plus longue; il y a un entostyle et un léger cingulum antéro-lingual.

La p2 est étroite et allongée; le protoconide est très élevé; le paraconide est très petit et bien formé, suivi antéro-lingualement par une petite lame représentant le parastylide; l'hypoconide est en position centrale, trois petites crêtes en descendent, une labialement, une distalement et la dernière postéro-labialement; à l'extrémité de cette dernière se dresse un petit hypoconulide.

La p4 et la m3 d'Estrepouy ont les caractéristiques morphologiques et les dimensions de la p4 et de la $\mathrm{m} 3$ de la mandibuletype de l'espèce (Ginsburg, 1971).

Famille Palaeomerycidae Lydekker, 1883

Genre Oriomeryx Ginssburg, 1985

Oriomeryx willii Ginsburg, 1985

Matériel: calcanéum g privé du manubrium (GER 750. $\mathrm{MNHN})$. DT $=23,3$; DAP $=30,5$. Figs. 9A-E.

Discussion: cette moitié antérieure de calcanéum est parfaitement bien conservée; elle est morphologiquement identique aux calcanéums d'Artenay, mais plus petite. Elle s'accorde parfaitement pour la taille avec un astragale et un cubo-naviculaire de Saint-Vincent-Lamontjoie ainsi qu'à un astragale de Chilleursaux-Bois. Nous rapporterons donc ce calcanéum au seul Palaeomerycidae connu dans la MN3a, Oriomeryx willii.

Famille Lagomerycidae Pilgrim, 1941

Genre Lagomeryx Roger, 1904

Lagomeryx parvulus (Roger, 1898)

1930 - Lagomeryx parvulus - Roman \& Viret p. 589

1934 - Lagomeryx parvulus - Roman \& Viret p. 53-54, pl. V, fig. 29

Matériel: m3g (FSL 320 154, figurée in Roman \& Viret (1934), pl.V, fig. 29) = 9,5 x 5. Figs 10 A-B-; m3g (coll. B $)=$ 8,7 x 4,7; M1/2d (Bâle, GB 994) = 6,5 x 7,8; M1/2d (Bâle, GB 996 $)=5,75 \times 6,9 ;$ M1/2g $($ Bâle, GB 83) $=6,2 \times 6,95$

Discussion: toutes ces dents sont identiques morphologiquement et dimensionnellement à leurs équivalentes du riche matériel de Lagomeryx parvulus d'Artenay.

Famille Cervidae Goldfuss, 1820

Genre Procervulus Gaudry, 1877

Procervulus praelucidus (Obergfell, 1957)

1930 - Amphitragulus sp. - Roman \& Viret p. 589

1934 - Amphitragulus aurelianensis Mayet: Roman \& Viret, pl. VII, fig. 12.

Matériel: p4 g (FSL 320.196), figurée in Roman \& Viret (1934), pl.VII, fig. 12) = 9,2x 5,4; P2g (coll. H) = 8,74 x 5,16; P4d $($ Bâle, GB 91) = 6,85 x 8,6; M3d (Bâle, GB 90) = $10 \times 1$ 1,5; M3d(coll. H) = 11,6 x 13,56; i1d (coll. H) = 5,95 x 3,4; M2g (GER 752. MNHN) muraille labiale. Fig. 11; p2d (FSL $320.336)=6,45 \times 2,2 ;$ m1d, très usée $($ coll. $\mathrm{H})=(8,8) \times 7,7$; Fragment de bois (GER 753. MNHN) Fig. 12
Discussion: toutes les dents ont des dimensions compatibles avec celles de la population de Procervulus praelucidus de Wintershof-West, qui est la population-type de l'espèce.

\section{Conclusion. Liste faunique}

Amphicyon lathanicus Ginsburg, 2000

Cynelos helbingi (Dehm, 1950)

Plithocyon bruneti Ginsburg, 1980

Hemicyon gargan Ginsburg et Morales, 1998

Palaeogale hyaenoides Dehm, 1950

Semigenetta elegans Dehm, 1950

Pseudaelurus turnauensis (Hoernes, 1882)

Anchitherium aurelianense (Cuvier, 1825)

Protaceratherium minutum (Cuvier, 1822)

Diaceratherium cf. aurelianense (Nouel, 1866)

Aureliachoerus aurelianensis (Stehlin, 1899)

Xenohyus venitor Ginsburg, 1980

Cainotherium aff. lintillae Baudelot et Crouzel, 1974

Andegameryx andegaviensis Ginsburg, 1971

Oriomeryx willii Ginsburg, 1985

Lagomeryx parvulus (Roger, 1898)

Procervulus praelucidus (Obergfell, 1957)

\section{Paleoenvironnement}

Mammifères variés et donnent une bonne représentation du panorama faunique au début des grands changements du Burdigalien. Ils montrent une certaine différenciation géographique entre Allemagne et France (voir Espagne). Artiodactyles: climat chaud et humide ou bien humide par saisons, végétation toujours verte, très boisée.

\section{Références}

Abel, O. (1910). Kritiche Untersuchungen über die paläogenen Rhinocerotiden Europas. Abhndlungen der K. K. geologisch. Reicsanstalt, 20: 1-52.

Abusch-Sievert, S. (1983). Gebissmorphologische Untersuchunden an eurasiatischen Anchitherien (Equidae, Mammalia) unter besonderer Berüksichtigung der Fundstelle Sandelzhausen. Courier Forschungsinstitut Senckenberg, 62: 1·361.

Baudelot, S. \& Crouzel, F. (1974). La faune burdigalienne des gisements d'Espira-du-Conflent (Pyrénees Orientales). Bulletin de la Societé d'Histoire Naturelle de Tolouse, 110: 311-326.

Blainville, M.De. (1937). Rapport sur un nouvel envoi de fossiles provenant du dépót de Sansan. Compte rendu des Seances de l'Academie des Sciences, 5: 417-427. 
Bowdich, T.E. (1821). An Analysis of the Natural Classifications of Mammalia for the Use of Students and Travelers. J. Smith, Paris, 115 pp.

Bravard A. (1828). Monographie de la montagne de Perrier près d'Issoire (Puy-de-Dôme) et de deux espèces du genre Felis, découvert dans l'une de ces couches d'alluvion. Paris: 1-147.

Baudelot S. \& Crouzel F. (1974). La faune burdigalienne des gisements d'Espira-du-Conflent (Pyrénées-Orientales). Bulletin de la Societé d'Histoire naturelle de Toulouse, 110 (3-4): 311-326.

Collier, A. \& Guex, H. (1977). Un crâne d'Aureliachoerus aurelianensis (Mammalia, Artiodactyla) dans le Burdigalien de La Romieu (Gers). Bulletin de la Société Archéologique et Historique du Gers, Auch: 463-470.

Cope, E.D. (1881). The Classification and Phylogeny of the Artiodactyla. Proceedings of the American Philosophical Society, 24: 377-400.

Cuvier G. (1824). Recherches sur les ossemens fossiles, où l'on rétablit les caractères de plusieurs animaux dont les revolutions du globe ont détruit les espèces. 2ème édition. Paris.

Cuvier, G. (1825). Recherches sur les ossements fossiles, 3e édit. 1, 412 p.

Dehm, R. (1950). Die Raubtiere aus dem Mittel-Miocän (Burdigalium) von Wintershof-West bei Eichstätt in Bayern. Abhandlungen der Bayerischen Akademie der Wissenschaften, Mathematischnaturwissenschaftliche Klasse NF, 58, 1-141.

Dietrich, W.O. (1931). Neue Nashornreste aus Schwaben (Diaceratherium tomerdingensis n. g. n. sp.). Zeitschrift für Saügetierkunde, 6: 203-220.

Fischer von Waldheim, G. (1813). Zoognosia tabulis synopticis illustrata. Moscow, Ncolai Sergeidis Vsevolozsky, vol. 2, 605 pp.

Gaudry, A. (1877). Les enchaînements du monde animal dans les temps geologiques: Mammiféres tertiaries. Savy, Paris, 293 pp.

Gervais, P. (1848-1952). Zoologie et paléontologie francais (animaux vertébrés) ou nouvelles recherches sur les animaux vivants et fossils de la France. Bertrand, Paris $271 \mathrm{pp}$.

Ginsburg, L. (1955). De la subdivision du genre Hemicyon Lartet (Carnassier du Miocène). Bulletin de la Société géologique de France, 6e sér., 5: 85-99, 2 fig.; Paris

Ginsburg, L. (1971). Un ruminant nouveau dans les faluns miocènes de la Touraine et de l'Anjou. Bulletin du Musée National d'Histoire Natural de Paris, 42: 996-1002.

Ginsburg, L. (1974). Les faunes de mammifères burdigaliens et vindoboniens des bassins de la Loire et de la Garonne. V Congrès du Néogène méditerranéen. Mémoires du Bureau de recherches géologiques et minières (BRGM), 78 (1), pp. 153-167.

Ginsburg, L. (1974). Les Tayassuidés des phosphorites du Quercy. Palaeovertebrata, 6: 55-85.

Ginsburg, L. (1977). Cynelos lemanensis (Pomel), carnivore ursidé de l'Aquitanien d'Europe. Annales de Paléontologie (Vertébrés), 63 (1): 57-104.
Ginsburg, L. (1980). Plithocyon bruneti nov. sp. Hemicyoninae (Ursidae, Carnivora, Mammalia) du Miocène de France. Comptes Rendus sommaires de la Societé Géologique de France, 6:232-235.

Ginsburg, L. (1980). Xenohyus venitor, Suidé nouveau (Mammalia, Artiodactyla) du Miocène inférieur de France. Geobios, 13: 861-877.

Ginsburg, L. (1985). Systématique et evolution du genre miocène Palaeomeryx (Artiodactyla, Giraffoidea) en Europe. Comptes Rendus de l'Académie des Sciences (IIa), 301 (14): (1075)-(1078).

Ginsburg, L. (1990). The faunas and stratigraphical subdivisions of the Orleanian in the Loire Basin (France). In: European Neogene Mammal Chronology (Lindsay, E.H.; Fahlbusch, V. \& Mein, P., eds.), NATQ ASI Series. Plenum Press, New York and London, 157176.

Ginsburg, L. (2000). Les espèces du genre Amphicyon et un Amphicyonidae (Carnivora, Mammalia) nouveau des faluns miocène d'Anjou. Symbioses, 3: 35-40.

Ginsburg L.; Huin, J. \& Locher, J.-P. (1985). Les Artiodactyles sélénodontes du Miocène inférieur des Beilleaux à Savigné-sur-Lathan (Indre-et-Loire). Bulletin du Muséum national d'Histoire naturelle 4e sér., 7C (4): 285-303.

Ginsburg, L. \& Morales, J. (1989). Les Ruminants du Miocène de Bézian à La Romieu (Gers). Bulletin du Muséum National d'Histoire Naturelle, 4C, 11: 201-231.

Ginsburg L. \& Morales, J. (1998). Les Hemicyoninae (Ursidae, Carnivora, Mammalia) et les formes apparentées du Miocène inférieur et moyen d'Europe occidentale. Annales de Paléontologie, 84 (1): 71-123. doi:10.1016/S0753-3969(98)80003-7

Goldfuss, G.A. (1820). Handbuch der Zoologie. Nürnberg, J.L. Schrag, 2 vols, 745 pp.

Gray, J.E. (1821). On the natural arrangement of vertebrose animals. London Medical Repository, 15 (1): 296-310.

Haeckel, L, E. (1866). Generelle Morphologie der Organismen. Volume 2. Georg Reimer, Berlin, $462 \mathrm{pp}$.

Hernández-Fernández, M.; Salesa, M.J.; Sánchez, I.M. \& Morales, J. (2003). Paleoecología del género Anchitherium von Meyer, (1834) (Equidae, Perissodactyla, Mammalia) en España: evidencias a partir de las faunas de macromamíferos. Coloquios de Paleontología, Vol. Extra., 1: 253-280.

Hoernes, R. (1882). Säugethier-Reste aus der Braunkohle von Göriach bei Turnau in Steiermark. Jahrbuch der kaiserlich-königlichen Geologischen Reichsanstalt, 32.

Lartet, E. (1837). Blainville, M.De. (1937), Rapport sur un nouvel envoi de fossiles provenant du dépót de Sansan. Compte rendu des Seances de l'Academie des Sciences, 5: 417-427.

Lartet, E. (1851). Notice sur la Colline de Sansan, suivi d'une récapitulation des diverses espèces d'animaux vertébrés fossiles, trouvés soit à Sansan dans d'autres gisements du terrain teritiare miocène dans le bassin sous-pyrénéen. J.A. Portes, Auch, 45 pp.

Lydekker, R. (1883). Indian Tertiary and post-Tertiary Vertebrata: Siwalik selenodont Suina. Records of the 
Geological Survey of India, Pal. Indica, 5(10): 143177.

Mayet, L. (1908): Etude des mammifères miocènes des sables de l'Orléanais et des Faluns de la Touraine. Annales de l'Université de Lyon, 1: 1-321.

Meyer, H. von (1834). Die fossilen Zähne und Knochen und ihre Ablagerung in der Gegend von Georgensgmünd in Bayern. J.D. Verlag Sauerländer, Frankfurt, $176 \mathrm{pp}$.

Meyer, H. von (1844). Die fossilen Knochen aus dem Tertiar-Gebilde des Cerro de San Isidro bei Madrid. Neues Jahrbuch für Mineralogie, Geognosie, Geologie und Peterfaktenkunde, 289-310.

Meyer, H. von (1846). (1846): Mitteilungen an Prof. Bronn gerichtet (Brief). - Neues Jahrbuch für Mineralogie, Geologie und Paläontologie, 462-476.

Nouel, A. (1866): Mémoire sur un nouveau Rhinocéros fossile. Mémoires de la Société Agricole et Scientifique, Orléans, 7: 1-13.

Obergfell, F.A. (1957). Vergleichende Untersuchungen an Dentitionen und Dentale altburdigaler Cerviden von Wintersshof-West in Bayer und rezenter Cerviden (eine Phylogenetische Studie). Palaeontographica, 103: 71-166.

Owen, R. (1848), Description of Teeth and portions of Jaws of two extinct Anthracotherioid Quadrupeds (Hyopotamus vectianus and Hyop. Bovinus) discovered by Marchioness of Hasting in the Eocene Deposits on the N.W. coast of the Isle of Wight: with an attempt to develop Cuvier's idea of the Classification of Pachyderms by the Number of their Toes. Quaterly Journal of the Geological Society of London, 4: 103-141.
Palmer, T.S. (1897): Notes on the nomenclature of four genera of tropical American mammals. Proceedings of the Biological Society Washington, 11: 173-174.

Pilgrim, E.G. (1941). The ddispersal of the Artiodactyla. Biological Reviews, 16: 134-163.

Répelin, J. (1917). Etudes paléontologiques dans le sudouest de la France (Mammifères). Les Rhinocérotidés de l'Aquitanien supérieur de l'Agenais (Laugnac). Annales du Muséum d'Histoire Naturelle de Marseille, 16: 1-47.

Roger, O. (1898). Wirbeltierreste aus dem Dinotheriesande bayerisch-schwäbischen Hochebene. Bericht Naturwissenschafhichen Vereins für Schawaben und Neuburg (a.v.), 33: 1-46

Roger, O. (1904). Wirbeltierreste aus dem Obermiocän der bayerisch-schwäbischen Hochebene. Bericht Naturwissenschafhichen Vereins für Schawaben und Neuburg (a.v.), 36: 3-22.

Roman, F.\& Viret, J. (1930). Le Miocène continental de l'Armagnac et le gisement burdigalien de La Romieu (Gers). Société Géologique de France, Livre Jubilaire 2: 577-604.

Roman, F. \& Viret, J. (1934). La faune de mammifères du Burdigalien de La Romieu (Gers). Mémoires de la Société Géologique de France (n.s.), 21: 5-67.

Sthelin, H.G. (1899)/(1900). Ueber die Geschchte des Suidengebisses. Abhndlungen der schweizerischen paläontologischen Gesellschaft, 26 [first part: (1899)]: 1-336; 27 [second part: (1900)]: 337-527.

Recibido el 20 de junio de 2011 Aceptado el 29 de agosto de 2011 